\title{
Primary Neoplasms of the Heart. Clinical and Histological Presentation of 50 Cases
}

\author{
Fábio Fernandes, Helena N. Soufen, Barbara M. lanni, Edmundo Arteaga, Félix J. A. Ramires, \\ Charles Mady
}

São Paulo, SP - Brazil

Objective - To analyze clinical and histologic findings of 50 patients with primary neoplams of the heart in a tertiary referral center.

Methods - From 1980 to 1998, we retrospectively analyzed 50 patients, 32 of whom were females, whose ages ranged from 9 to 73 years (mean age $=44.16 \pm 18$ years).

Results-Most tumors were located in the left side of the heart (72\%), myxoma being the most common (84\%) histologic type. The other histologic types found were as follows: fibroma (4\%), lipoma (2\%), rhabdomyosarcoma $(2 \%)$, hemangioma (2\%), sarcoma (2\%), angiosarcoma $(2 \%)$, and lymphoma (2\%). Diagnosis was established by echocardiography in $94 \%$ of the cases. Clinical findings were as follows: dyspnea (36\%), weight loss (20\%), palpitations (18\%), chest pain (16\%), fever (8\%), and arthralgia (6\%). All patients with thromboembolic phenomena $(10 \%)$ had left atrial myxoma. Approximately $20 \%$ of the patients were asymptomatic at the initial clinical assessment.

Conclusion - Primary cardiac tumors are a rare entity with diverse clinical and histologic findings, requiring, therefore, a high level of clinical suspicion.

Key words: cardiac tumor, myxoma
In recent decades, with the introduction of new imaging methods in cardiology, particularly echocardiography, an increase in the diagnosis of cardiac tumors has occurred, which was formerly possible only during surgery or autopsy. The clinical findings of cardiac tumors vary. They may mimic either cardiac or systemic diseases, and, therefore, should be considered in the differential diagnosis of distinct heart diseases. In addition, as surgical therapy is curative in most cases, an early diagnosis is required to prevent complications.

Neoplasms may involve the heart in a primary or secondary way, and no consensus exists in regard to their classification. Secondary tumors are 20 to 40 times more frequent than primary ones, among which the benign are 3 times more frequent than the malignant ones ${ }^{1,2}$. Neoplasm of the heart is a rare entity, with an incidence ranging from $0.0017 \%$ to $0.28 \%$. It may involve the endocardium, myocardium, or epicardium.

This study aims to report the clinical characteristics and histologic types of primary cardiac tumors in patients referred to a tertiary service.

\section{Methods}

We analyzed retrospectively 50 patients ( 32 females) with ages ranging from 9 to 73 years (mean of $44.6 \pm 18$ ) who were diagnosed with primary cardiac tumors at the Instituto do Coração (InCor) of the Hospital das Clínicas of FMUSP, from 1980 to 1998 . The diagnosis was established by echocardiography in 47 patients, by magnetic resonance in 2 patients, and during surgery in 1 patient (myocardial revascularization).

Instituto do Coração do Hospital das Clínicas - FMUSP

Mailing address: Fábio Fernandes - InCor - Unidade Clínica de Cardiopatias Gerais - Av. Dr. Enéas C. Aguiar, 44 - 05403-900 - São Paulo, SP, Brazil - E-mail: car_fabio@incor.usp.br

English version by Stela Maris C. Gandour

\section{Results}

In our study, the cardiac tumors were benign in $92 \%$ of the patients. Their preferred site was the left side of the heart (72\%), 66\% being in the left atrium, $6 \%$ in the left ventricle, $22 \%$ in the right atrium, $4 \%$ in the right ventricle,
\end{abstract}


and $2 \%$ in the interventricular septum. In 1 patient, a rhabdomyosarcoma was located in the right atrium and left ventricle, and in another patient a low-grade sarcoma was located in the left atrium and ventricle. The most common histologic type was the cardiac myxoma (fig. 1 and 2), and the remaining histologic types are listed in table I. The duration of clinical symptoms varied from 1 day to 60 months, with a mean of 15.48 months.

In regard to clinical findings (Table II), the most frequent symptom was dyspnea (36\%), followed by other

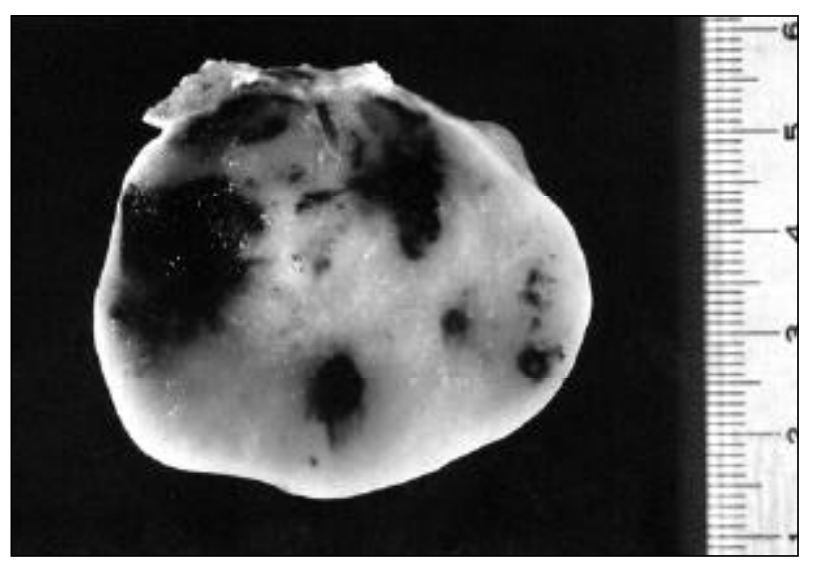

Fig. 1 - Left atrial myxoma - gross appearance. Note the gelatinous appearance of the tumor with hemorrhagic foci.

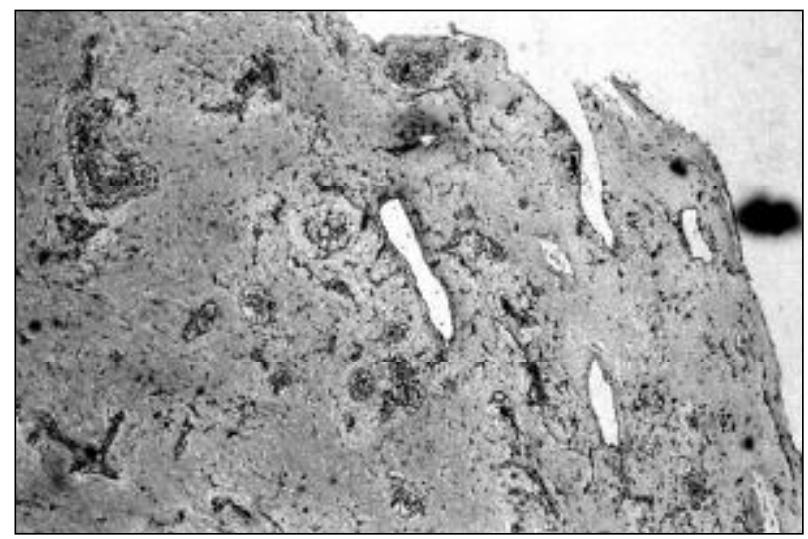

Fig. 2 - Left atrial myxoma - histologic appearance (HE 10X). Note the large amount of myxoid matrix (stained in pink).

\begin{tabular}{|lcc|}
\hline \multicolumn{2}{|c|}{ Table I - Histologic types } \\
\hline & $\mathrm{N}$ & $\%$ \\
\hline Left ventricular myxoma & 1 & 2 \\
Left atrial myxoma & 33 & 66 \\
Right atrial myxoma & 22 & 16 \\
Fibroma & 2 & 4 \\
Fibroma of Left ventricle & 1 & 2 \\
Fibroma of Septum & 1 & 2 \\
Lipoma & 1 & 2 \\
Hemangioma & 1 & 2 \\
Angiosarcoma & 1 & 2 \\
Low-grade sarcoma & 1 & 2 \\
Lymphoma & 1 & 2 \\
\hline
\end{tabular}

symptoms of heart failure in $28 \%$ of the patients. Other cardiac symptoms reported include the following: palpitations (18\%), chest pain (16\%), embolism (10\%), and near syncope $(2 \%)$. Embolism occurred to the cerebral area resulting in cerebral stroke $(6 \%)$, to the heart resulting in myocardial infarction (2\%), and to the ophthalmic artery resulting in amaurosis (2\%). Extracardiac symptoms were present in $36 \%$ of the patients, the most frequently reported being weight loss in $20 \%$, followed by fever (8\%), arthralgias (6\%), and chronic anemia (2\%). Approximately $20 \%$ of the patients were asymptomatic at the initial clinical evaluation.

On physical examination, auscultation revealed a mitral diastolic murmur in $34 \%$ of the patients, a mitral systolic murmur in $14 \%$ of the patients, and a tricuspid systolic murmur in $2 \%$ of the patients.

From the 50patients with cardiac tumors, $40(80 \%)$ were operated upon, and $8(16 \%)$ died during the clinical evolution (Table III). The deaths were secondary to acute pulmonary edema and heart failure $(4 \%)$, to cardiogenic shock (2\%), pulmonary hemorrhage $(2 \%)$, disseminated sarcomatosis (4\%), sepsis and pulmonary infection (2\%), cerebral metastasis to the pleura and pericardium (2\%), and to embolism of tumor fragments $(2 \%)$ causing acute myocardial infarction. From the total deaths, $6 \%$ of the patients died prior to surgical treatment, $4 \%$ because of refractory acute pulmonary edema, and $2 \%$ because of acute myocardial infarction. Surgical deaths were secondary to sepsis (2\%), cardiogenic shock (2\%), and pulmonary hemorrhage (2\%).

\section{Discussion}

In the 50 cardiac tumors of our case series, $46(92 \%)$ were benign, cardiac myxoma being the most frequent one $(84 \%)$. Myxoma is the most prevalent cardiac tumor in the

\begin{tabular}{|lc|}
\hline \multicolumn{2}{|c|}{ Table II - Clinical findings } \\
\hline Cardiac symptoms & $\%$ \\
Dyspnea & 36 \\
Heart failure & 28 \\
Palpitations & 18 \\
Chest pain & 16 \\
Embolism & 10 \\
Near syncope & 2 \\
Extracardiac symptoms & \\
Weight loss & 20 \\
Fever & 8 \\
Arthralgias & 6 \\
Chronic anemia / disease & 2 \\
\hline
\end{tabular}

\begin{tabular}{|lc|}
\hline \multicolumn{2}{|c|}{ Table III - Classification of the deaths } \\
\hline Congestive heart failure & $4 \%$ \\
Disseminated sarcomatosis & $4 \%$ \\
Pulmonary hemorrhage & $2 \%$ \\
Acute myocardial infarction & $2 \%$ \\
Cardiogenic shock & $2 \%$ \\
Septicemia & $2 \%$ \\
Total & $16 \%$ \\
\hline
\end{tabular}


adult population, predominating in the female sex, with a gelatinous and transparent gross appearance.

Myxomas are usually single tumors, preferably located in the left atrium and inserted close to the oval fossa. They are considered benign tumors, even though they may behave in a malignant way with local relapse, invasion of the thoracic wall, and embolism.

Symptoms result from the size of the neoplasm (usually $70 \mathrm{~g}$ ), which may cause obstruction of the cardiac chambers and valve rings, or they may also be consequent to embolism of tumor fragments occurring later ${ }^{2}$, because of the gelatinous characteristic of the tumor, or may even result from formation of thrombi. In our case series, embolism occurred in patients with left atrial myxoma, being the presentation symptom in $10 \%$ of them. Among our patients, we had 3 strokes, 1 myocardial infarction, and 1 amaurosis. One case reported in the literature ${ }^{3}$ shows a possible association of left atrial myxoma and acute myocardial infarction of the inferior wall. In our study, the myocardial infarction was in the anterior wall and the patient died. On anatomicopathological study, fragments of the tumor were found in the coronary artery involved. In the medical literature, the association between myxoma and myocardial infarction has not been frequently reported, approximately less than $0.1 \%{ }^{3}$.

In a few cases, myxoma may simulate systemic diseases with symptoms such as fever, cachexia, arthralgia, Raynaud's phenomenon, rash, and anemia, which are attributed to the production of certain cytokines, such as interleukin- $6{ }^{4}$. In our case series, systemic manifestations were present in $36 \%$ of the patients, 1 patient being on corticoids due to rheumatoid disease, and another patient being on antibiotics due to the diagnosis of bacterial endocarditis.

Cardiac tumors may obstruct the cardiac chambers mimicking either myocardial or valvular diseases. Obstruction of the right chambers simulates constrictive pericarditis, tricuspid stenosis, and thromboembolism. Signs and symptoms of right ventricular tumors are similar to those of pulmonary stenosis. On the other hand, left atrial tumors are clinically similar to mitral stenosis. Symptoms reported in tumors of the left ventricle and septum are as follows: sudden death, dyspnea, syncope, and precordial pain ${ }^{5}$. A symptomatic characteristic suggesting the presence of neoplasm of the heart is change of symptoms according to certain bodily positions.

On clinical examination, left atrial tumors are similar to mitral stenosis. The following points help in the clinical differentiation: influence of position, absence of the opening click and of the split of the first heart sound. In other cases, the audible murmur may be of mitral regurgitation, being secondary to the chronic tension of the valve or consequent to incomplete closing during systole. In the case of right atrial tumors, auscultation is characterized by a split of the first heart sound, but the components of the second heart sound tend to fuse. This phenomenon results from an increase in right atrial pressure.

Another cardiac symptom frequently reported by our patients was the presence of arrhythmias (18\%), which may result from enlargement of the cardiac chambers or may be secondary to invasion of the myocardium or the conducting tissue by the tumor ${ }^{6}$.

Chest pain was present in $16 \%$ of the patients and had an atypical characteristic. In some cases, the pain may have characteristics typical of coronary heart disease, which may result from tumoral invasion or, as in 1 of our cases, from coronary embolism. This must be included in a differential diagnosis considered in young individuals with acute myocardial infarction. Myxomas and malignant tumors are rare in the pediatric age group ${ }^{7}$.

Other tumors of the connective tissue, such as lipomas and fibromas, occur in the heart, as in other parts of the body, being considered very rare. Their symptoms are associated with obstruction in the cardiac chambers ${ }^{8}$.

Among the malignant tumors of the heart, sarcomas are the most frequent. In our case series we had 1 low-grade sarcoma, 1 rhabdomyosarcoma, and 1 angiosarcoma. The angiosarcoma has a poor prognosis. It is located preferably in the right atrium and is often associated with a bloody pericardial effusion, which may be confounded with tuberculous pericarditis. Metastases are reported in this type of tumor; in our case series, 1 patient had cerebral metastases ${ }^{9}$.

Treatment of primary tumors of the heart is exeresis, to avoid the complications of the disease, such as obstruction or embolism. Literature reports show that $8 \%$ of the patients with heart tumors died while awaiting surgical treatment ${ }^{10}$.In our study, 3 patients died without surgery, 2 due to acute pulmonary edema, and one because of myocardial infarction.

In conclusion, primary tumors of the heart are rare entities that may simulate either cardiac or systemic diseases, which implies the need for a diagnosis of high clinical suspicion.

\section{References}

1. Shackell M, Mitko A, Williams PL, Stton GC. Angiosarcoma of the heart. Br Heart J 1979; 41: 498-503.

2. Bjessmo S, Ivert T. Cardiac myxoma: 40 years. Experience in 63 patients. Ann Thorac Surg 1997; 63: 697-700.

3. Meira EBS, Ho CL, Santos-Filho MM, Pimenta J. Mixoma de átrio esquerdo provocando infarto agudo miocárdio. Arq Bras Cardiol 1996; 67: 347-9.

4. Lehmam KL, Prozan GB, ULLyot D. Atrial myxoma presenting as acute myocardial infarction. Am Heart J 1985; 110: 1293-5.

5. Colucci WS, Schoen FJ, Braunwald E. Primary tumors of the heart. In: Brauwald E - Heart Disease. A Textbook of Cardiovascular Medicine. $5^{\text {the }}$ d.. Philadelphia: WB Saunders, 1998: 1464-78.
6. Goodwin JF. Symposium on cardiac tumors. The spectrum of cardiac tumors. Am J Cardiol 1968 21: 307-14

7. Roberts W C. Primary and secondary neoplasm of the heart. Am J Cardiol 1997; 80: 671-82.

8. Almeida E C, Leite MSB, Silva Ma, Rassi L. Rabdomioma de ventrículo direito causando estenose pulmonar. Arq Bras Cardiol 1993; 60: 417-9

9. Ianni BM, Mady C, Barretto ACP, et al. Angiossarcoma de coração. Relato de caso. Arq Bras Cardiol 1986; 47: 211-15.

10. Attar S, Lee Y, Singleton R, Scherlis L, David R, MeLanghlins JS. Cardiac Myxoma. Ann Thorac Surg 1980; 29: 397- 405. 